\title{
Management of nasopharyngeal angiofibroma in a 72-year-old male through a sublabial and buccolabial incision approach: A case report and literature review
}

\author{
HUAN-KANG ZHANG, JING-JING WANG, ZHUO-FU LIU and DE-HUI WANG \\ Department of Otolaryngology, Fudan University Affiliated Eye, Ear, Nose and Throat Hospital, Shanghai 200031, P.R. China
}

Received July 12, 2014; Accepted March 24, 2015

DOI: $10.3892 / \mathrm{ol} .2015 .3410$

\begin{abstract}
Nasopharyngeal angiofibroma (NA) primarily affects adolescent males. The incidence of NA in the elderly, however, is extremely low. The present study describes a 72-year-old male with NA that presented with typical symptoms and radiological findings. The location and extension of the tumor was too lateral and inferior to be effectively resected by an endoscopic approach. Therefore, a combination of endoscopic and open approaches was considered. The tumor was completely resected using an image-guided endoscopic-assisted sublabial and buccolabial incision approach. The post-operative histopathological and immunohistochemical analysis confirmed the diagnosis of NA. The present study demonstrated the occurrence of NA in the elderly, and highlighted the potential of an image-guided endoscopic-assisted sublabial and buccolabial incision approach for the treatment of NA in the elderly.
\end{abstract}

\section{Introduction}

Nasopharyngeal angiofibroma(NA) is a rare, non-encapsulated and highly-vascularized benign tumor that primarily affects adolescent males (1,2). Extremely few studies have described NA in elderly individuals; in our previous report of 162 cases from 1995 to 2012, all patients were male subjects ranging in age from 8-41 years (mean, 17.5 years) (3). Although NA is a benign neoplasm histopathologically, it has a propensity for locally destructive growth with bone erosion, which can lead to life threatening complications, such as fatal epistaxis, and other complications, including facial swelling, proptosis, cranial neuropathy, and intraoperative massive hemorrhage $(1,4)$. In the majority of cases, patients present with symptoms of a painless, unilateral nasal obstruction and

Correspondence to: Professor De-Hui Wang, Department of Otolaryngology, Fudan University Affiliated Eye, Ear, Nose and Throat Hospital, Room 204, Building 10, 83 Fenyang Road, Shanghai 200031, P.R. China

E-mail: wangdehuient@sina.com

Key words: nasopharyngeal angiofibroma, aged, histopathology, immunohistochemistry epistaxis (4). Currently, surgery is the standard treatment for NA (5).

The present study reports the case of a 72-year-old male with NA that underwent a successful resection via an endoscopic-assisted sublabial and buccolabial incision approach (6). This case confirms the occurrence of NA in the elderly, and also highlights the potential of an endoscopic-assisted sublabial and buccolabial incision approach for the treatment of NA in elderly patients.

\section{Case report}

A 72-year-old male presented with continuous headaches, right nasal epistaxis, right nasal obstruction and a decreased sense of smell for three months. The patient was admitted to the First Affiliated Hospital of Medical School of Zhejiang University (Hangzhou, China) on March 29, 2013, three months after the initial onset of these symptoms. The contrast-enhanced computed tomography (CT) and magnetic resonance imaging (MRI) revealed a highly vascular nasopharyngeal mass, and the sphenomaxillary fossa was enlarged (Fig. 1A and B). Surgical resection was performed using a lateral rhinotomy approach. However, due to massive hemorrhage, the tumor was not completely removed. Histopathological examination of the excised lesion revealed the presence of angiofibroma. Five months after surgery, the patient presented with recurrent right spontaneous epistaxis and right nasal obstruction and was subsequently referred to the Fudan University Affiliated Eye, Ear, Nose and Throat Hospital (Shanghai, China) to receive additional treatment.

A physical examination at our hospital revealed a red mass in the right nasal cavity. Contrast-enhanced CT confirmed the presence of a large soft-tissue nasal and nasopharyngeal mass that extended into the right maxillary antrum and up towards the orbital floor, with destruction of the medial and lateral walls of the antrum, into the sphenoid sinus on the right side and continued dorsally to the right parapharyngeal space and infratemporal fossa (Fig. 1C).

An endoscopic-assisted sublabial and buccolabial incision approach was used to remove the tumor following pre-operative embolization. Using clamp forceps, the nasal component of the tumor was pushed down into the nasopharynx and drawn out through the oral cavity. In order to remove the infratemporal fossa extension of the NA, a sublabial and 
Table I. Three patients over the age of 70 diagnosed with nasopharyngeal angiofibroma.

\begin{tabular}{lccl}
\hline First author (ref.) & Gender & Age, years & \multicolumn{1}{c}{ Surgical approach } \\
\hline Conley et al (7) & Male & 79 & Transpalatine or lateral rhinotomy approach \\
Ewing and Shively (8) & Female & 71 & Right lateral rhinotomy and antrotomy \\
Shaheen (9) & Male & 70 & Moure's lateral rhinotomy incision \\
\hline
\end{tabular}
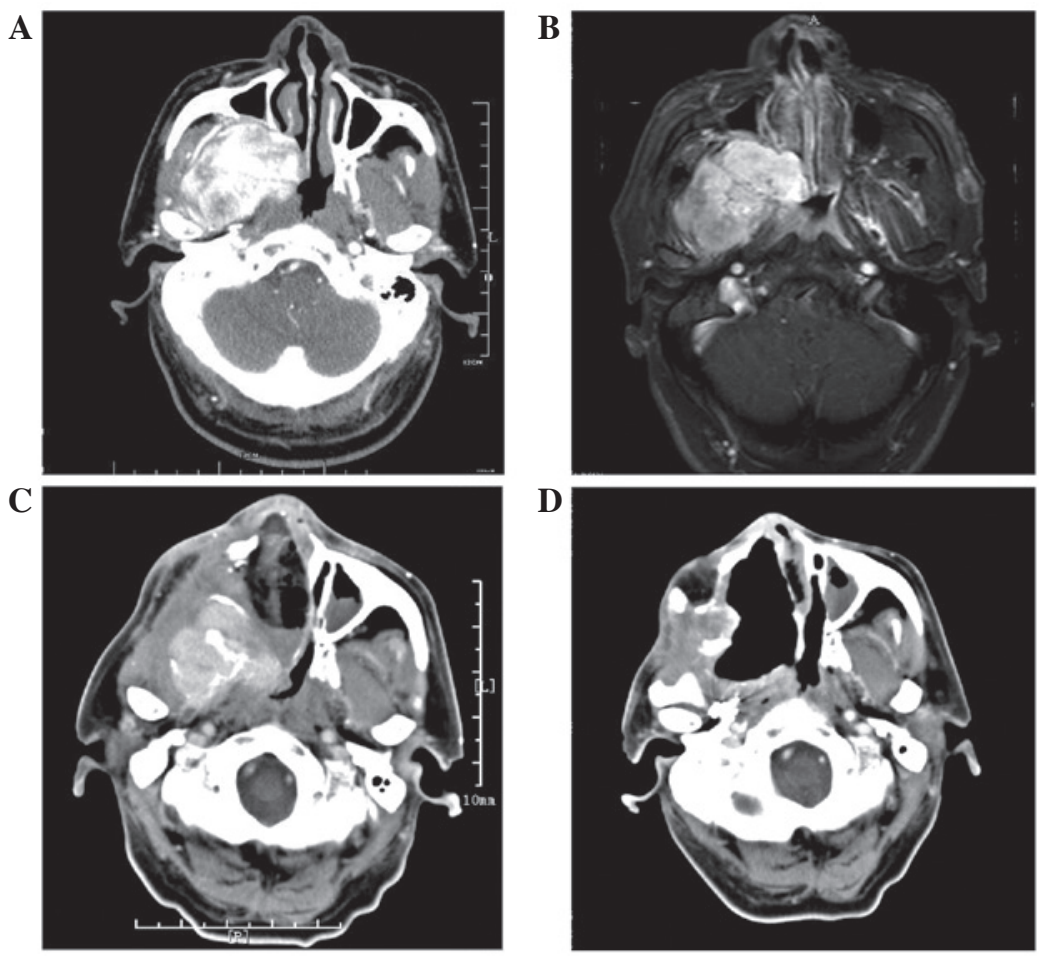

Figure 1. Radiological findings. (A) Pre-operative CT scan revealing a strongly enhancing, expanding mass originating in the nasopharynx and invading the right maxillary sinus, anterior and posterior ethmoid sinus, parapharyngeal space and infratemporal fossa. (B) Pre-operative T1-weighted axial magnetic resonance imaging revealing a strongly enhancing tumor following the administration of a contrast medium. (C) CT scan revealing the recurrence of the tumor five months after the lateral rhinotomy was performed. The large soft-tissue nasal-nasopharyngeal mass had extended into the right parapharyngeal space and infratemporal fossa. (D) CT scan revealing the complete removal of the tumor two weeks after a repeat endoscopic-assisted sublabial and buccolabial incision approach was performed. CT, computed tomography.

buccolabial incision, which extended from the first molar teeth to the maxillary tuberosity at the gingivobuccal sulcus, was made on the involved side. Due to the complex anatomy and high vascularity of the region, a blood loss of $\sim 2,800 \mathrm{ml}$ occurred during the surgery. The patient therefore received a blood transfusion of $400 \mathrm{ml}$ fresh frozen plasma and 4 units of packed red blood cells. The extensive hemorrhage also led to a poor surgical field. In order to prevent accidental injury, the surgery was terminated without further investigation. A contrast-enhanced CT scan was performed the next day to determine whether the tumor had been completely removed. The results revealed the presence of residual tumor in the right parapharyngeal space and infratemporal fossa. A day later, a repeat endoscopic-assisted sublabial and buccolabial incision approach was performed to remove the residual tumor. An image-guided navigation system (Medtronic, Louisville, KY, USA) was used for the localization of the residual tumor in the right parapharyngeal space and infratemporal fossa (Fig. 2). A contrast-enhanced CT scan performed 2 weeks subsequent to the procedure confirmed the complete removal of the tumor (Fig. 1D). The patient was followed up for 6 months, without exhibiting evidence of recurrence.

A hematoxylin and eosin-stained section revealed that the tumor was composed of a characteristic fibrous stroma in which the vascular channels were lined by flat endothelial cells (Fig. 3A). A Van-Gieson-stained section established that the tumor was mainly composed of collagen fibers, while elastic fibers were scarcely discernible (Fig. 3B). Immunohistochemically, the tumor was positive for the expression of cluster of differentiation (CD)31 (Fig. 3C), CD34 (Fig. 3D), CD68 (Fig. 3E), HHF35 (Fig. 3F), smooth muscle actin (Fig. 3G), and vimentin (Fig. 3H). The percentage of Ki-67-positive cells was 4\% (Fig. 3I). According to these findings, a diagnosis of angiofibroma was established.

This study was approved by the Ethics Committee of Fudan University Affiliated Eye, Ear, Nose and Throat Hospital, and written informed consent was obtained from the patient and the patient's family. 


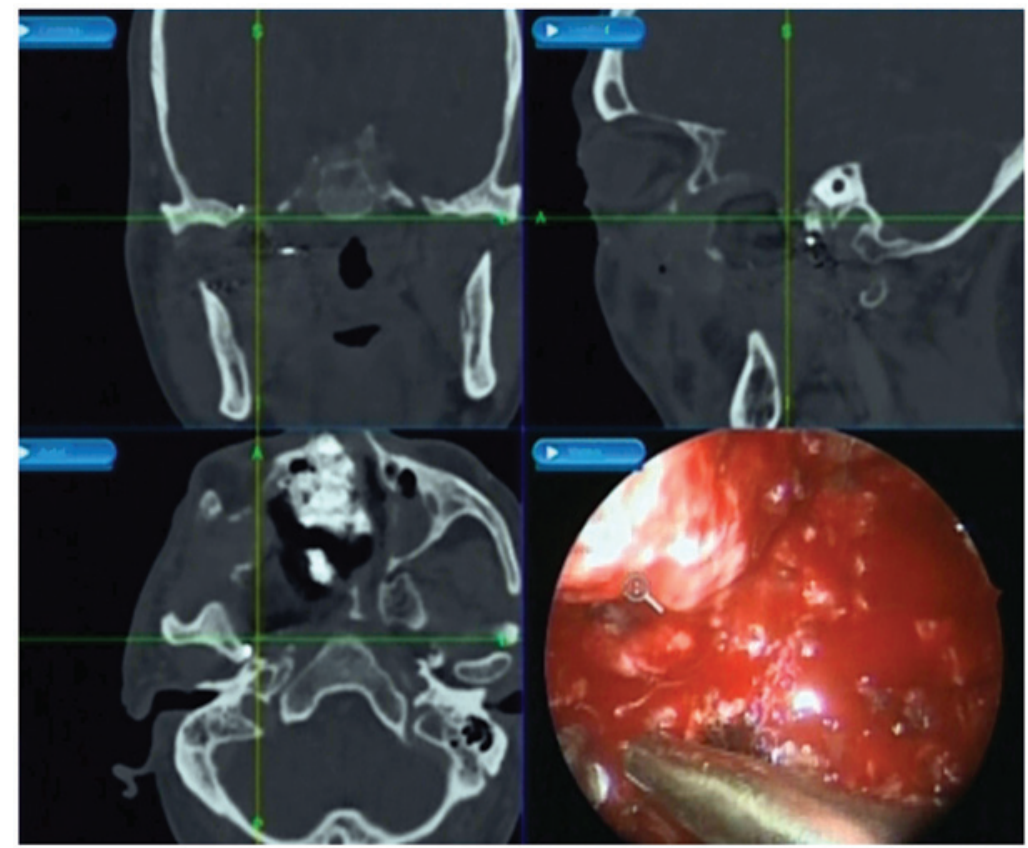

Figure 2. Revision surgery. A repeat endoscopic-assisted sublabial and buccolabial incision approach was performed in order to remove the residual tumor. An image-guided navigation system was used for the localization of the residual tumor in the right parapharyngeal space and infratemporal fossa.
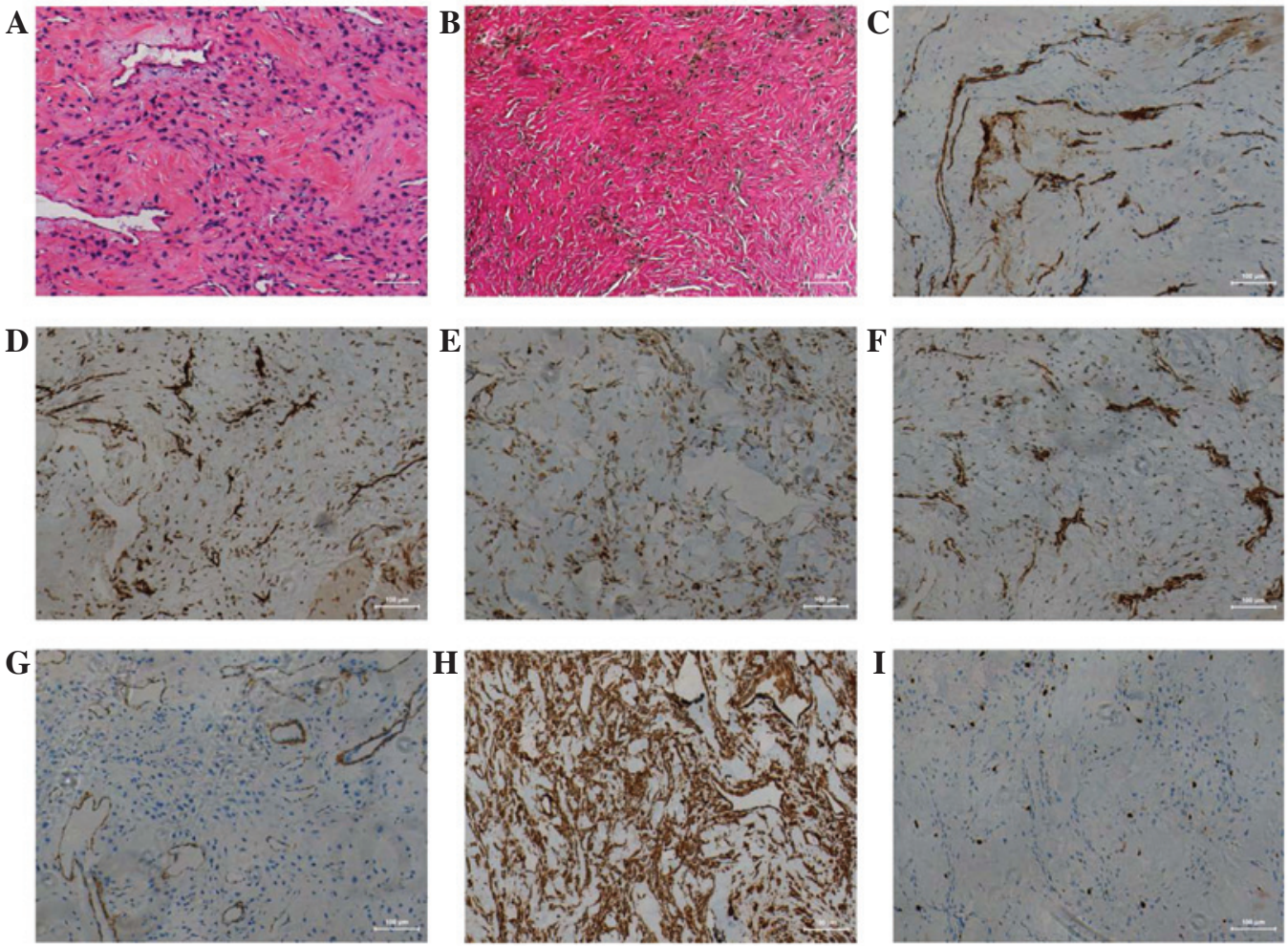

Figure 3. Results of the histopathological and immunohistochemical studies established a diagnosis of angiofibroma. (A) Hematoxylin and eosin-stained section revealing that the tumor was composed of microvascular embedded in fibrous tissue. (B) Van-Gieson-stained section revealing that the tumor was mainly composed of collagen fibers, while muscle fibres were scarcely discernible. Immunohistochemically, the tumor was positive for (C) CD31, (D) CD34, (E) CD68, (F) HHF35, (G) smooth muscle actin and (H) vimentin. (I) The percentage of Ki-67-positive cells was 4\%. Scale bar, 100 $\mu \mathrm{m}$. CD, cluster of differentiation.

\section{Discussion}

In general, NA primarily affects adolescent males, and is therefore considered to be a juvenile disease. However, NA has been observed in at least three patients over the age of 70 (Table I), consisting of a 79-year-old male (7), a 71-year-old female (8) and a 70-year-old male (9). These patients were treated by traditional open surgical approaches and the 
diagnoses were lacking in immunohistochemical evidence. According to Patrocinio et al (10), immunohistochemical analyses are required for the diagnosis of atypical NA cases, particularly those with atypical clinical manifestations. The immune profile is extremely useful during the process of pathological differential diagnosis.

The present study reported a rare case of NA in a 72-year-old male, which was confirmed by histopathological and immunohistochemical analysis. With the exception of age, the patient demonstrated typical presentation, clinical examination and radiological findings (Fig. 1). Histologically and immunohistochemically, the tumor was composed of proliferating vasculature in a fibrous stroma. The presence of red-colored regions following Van-Gieson staining and a strong positivity to vimentin indicated that the majority of the stromal cells were fibrocytes (11). Positive immunohistochemical staining for the endothelial markers CD31 and CD34 supported true endothelial differentiation (Fig. 3) (12). This typical presentation was of significant value for the diagnosis of NA.

The etiology and pathogenesis of NA remains unclear. It has been reported that no abnormalities in the serum levels of dihydrotestosterone, testosterone and estradiol-17 B are detected in NA patients (13). To a certain extent, the occurrence of NA in the elderly may support the hypothesis that serum androgen levels do not affect the development of NA, since low serum androgen levels are present in older men $(14,15)$.

Large NAs continue to present a considerable surgical challenge $(1,6)$. The optimal treatment for these tumors is gross total resection. However, open approaches require external and internal incisions, which results in extensive soft-tissue dissection and multiple osteotomies (3). Improvements in pre-operative assessment and preparation, in addition to operative techniques and instrumentation, may enable the endoscopic removal of the majority of juvenile NAs (16). If the location or extension of the tumor is too lateral or inferior to be effectively resected by an endoscopic approach, a combination of endoscopic and open approaches may be considered $(17,18)$. It has previously been reported that an endoscopic-assisted sublabial and buccolabial incision is an optional approach for the removal of an NA with extensive infratemporal fossa extension in youths (6). The present case confirmed that this modified surgery is well tolerated by the elderly. Due to a firm connection with surrounding structures, the tumor may break during surgery, leaving remnants in the surgical cavity, which poses a challenge for effective treatment. As a result of tumor extension and massive intraoperative hemorrhage, complete resection of the tumor was unsuccessful following the first endoscopic-assisted sublabial and buccolabial incision approach in the current case. Therefore, a revision surgery was considered. Image-guided navigation systems are useful for the localization of deep located tumors (19). In the present study, radiographical analysis revealed that the tumor had been completely resected following image-guided surgery. This counter example illustrated the value of the image-guided navigation system.

The present study confirmed the occurrence of NA in the elderly and highlighted the potential of an image-guided endoscopic-assisted sublabial and buccolabial incision approach for the treatment of NA in the elderly. In addition, the present study established that image-guided navigation systems are useful for the localization of deep NA lesions.

\section{Acknowledgements}

The authors would like to thank the patient for his contribution to this work. This work was supported by the National Natural Science Foundation of China (grant no. 81371077) and by Shanghai Municipal Hospitals' Rising and Leading Technology Program (grant no. SHDC12013121).

\section{References}

1. Sun XC, Wang DH, Yu HP, et al: Analysis of risk factors associated with recurrence of nasopharyngeal angiofibroma. J Otolaryngol Head Neck Surg 39: 56-61, 2010.

2. Boghani Z, Husain Q, Kanumuri VV, et al: Juvenile nasopharyngeal angiofibroma: A systematic review and comparison of endoscopic, endoscopic-assisted and open resection in 1047 cases. Laryngoscope 123: 859-869, 2013.

3. Huang Y, Liu Z, Wang J, et al: Surgical management of juvenile nasopharyngeal angiofibroma: Analysis of 162 cases from 1995 to 2012. Laryngoscope 124: 1942-1946, 2014

4. Tang IP, Shashinder S, Gopala Krishnan G and Narayanan P: Juvenile nasopharyngeal angiofibroma in a tertiary centre: Ten-year experience. Singapore Med J 50: 261-264, 2009.

5. Paris J, Guelfucci B, Moulin G, et al: Diagnosis and treatment of juvenile nasopharyngeal angiofibroma. Eur Arch Otorhinolaryngol 258: 120-124, 2001.

6. Sun XC, Li H, Liu ZF, et al: Endoscopic-assisted sublabial and buccolabial incision approach for juvenile nasopharyngeal angiofibroma with extensive infratemporal fossa extension. Int $\mathrm{J}$ Pediatr Otorhinolaryngol 76: 1501-1506, 2012.

7. Conley J, Healey WV, Blaugrund SM and Erzin KH: Nasopharyngeal angiofibroma in the juvenile. Surg Gynecol Obstet 126: 825-837, 1968.

8. Ewing JA and Shively EH: Angiofibroma: a rare case in an elderly female. Otolaryngol Head Neck Surg 89: 602-603, 1981.

9. Shaheen HB: Nasopharyngeal fibroma. J Laryngol Otol 45: 259-264, 1930

10. Patrocínio JA, Patrocínio LG, Borba BH, et al: Nasopharyngeal angiofibroma in an elderly woman. Am J Otolaryngol 26: 198-200, 2005.

11. Bornemann A and Schmalbruch H: Anti-vimentin staining in muscle pathology. Neuropathol Appl Neurobiol 19: 414-419, 2009.

12. Pusztaszeri MP, Seelentag $W$ and Bosman FT: Immunohistochemical expression of endothelial markers CD31, CD34, von Willebrand factor, and Fli-1 in normal human tissues. J Histochem Cytochem 54: 385-395, 2006.

13. Farag MM, Ghanimah SE, Ragaie A and Saleem TH: Hormonal receptors in juvenile nasopharyngeal angiofibroma. Laryngoscope 97: 208-211, 1987.

14. Ferrini RL and Barrett-Connor E: Sex hormones and age: a cross-sectional study of testosterone and estradiol and their bioavailable fractions in community-dwelling men. Am J Epidemiol 147: 750-754, 1998.

15. Laughlin GA, Barrett-Connor E and Bergstrom J: Low serum testosterone and mortality in older men. J Clin Endocrinol Metab 93: 68-75, 2008.

16. Douglas R and Wormald PJ: Endoscopic surgery for juvenile nasopharyngeal angiofibroma: Where are the limits? Curr Opin Otolaryngol Head Neck Surg 14: 1-5, 2006.

17. Khalifa MA and Ragab SM: Endoscopic assisted antral window approach for type III nasopharyngeal angiofibroma with infratemporal fossa extension. Int J Pediatr Otorhinolaryngol, 72: 1855-1860, 2008.

18. Gallia GL, Ramanathan M Jr, Blitz AM and Reh DD: Expanded endonasal endoscopic approach for resection of a juvenile nasopharyngeal angiofibroma with skull base involvement. J Clin Neurosci 17: 1423-1427, 2010.

19. Farhadi M, Jalessi M, Sharifi G, et al: Use of image guidance in endoscopic endonasal surgeries: A 5-year experience. B-ENT 7: 277-282, 2011. 